This is a post-peer-review, pre-copyedit version of a paper published in New Advances in Mechanisms, Mechanical Transmissions and Robotics. Mechanisms and Machine Science, vol 46. pp. 457-464 (2017) Springer, Cham. The final authenticated version is available online at: $\underline{\text { http://dx.doi.org/10.1007/978-3-319-45450-4_46 }}$

\title{
Kinematic Analysis of a Flexible Tensegrity Robot
}

\author{
O. Altuzarra ${ }^{1}$, M. Diez ${ }^{2}$, J. Corral ${ }^{3}$, F.J. Campa ${ }^{4}$ \\ ${ }^{1}$ University of the Basque Country UPV/EHU, Spain, e-mail: \\ oscar.altuzarra@ehu.eus \\ ${ }^{2}$ University of the Basque Country UPV/EHU, Spain, e-mail: mikel.diez@ehu.eus \\ ${ }^{3}$ University of the Basque Country UPV/EHU, Spain, e-mail: j.corral@ehu.eus \\ ${ }^{4}$ University of the Basque Country UPV/EHU, Spain, e-mail: fran.campa@ehu.eus
}

\begin{abstract}
.
In the field of parallel kinematics few designs use highly deformable elements to obtain the end effector movement. Most compliant mechanisms rely on notches or shape changes to simulate a standard kinematic joint. In this work a kinematic model of a simple parallel continuum mechanism that combines a deformable element and cable is presented. The kinematic model is used to study the workspace of the manipulator and is validated by experimental measurements of a prototype.
\end{abstract}

Key words: Parallel Continuum Robot, Compliant Mechanism, Kinematic Analysis, Experimental Mechanics.

\section{Introduction}

A new trend in parallel kinematic manipulators design is the use of ultradeformable elements in order to obtain the end effector movement [1,2]. One critical problem in the design process of this type of robots is the lack of available information, which usually is reduced to classical texts about nonlinear deformations [3]. Some MEMS (Micro Electro Mechanisms) [4, 5] do share the parallel morphology but lack the non-linear deformations that their macroscopic counterparts do suffer.

In this paper a two-degree of freedom parallel continuum robot is studied. The mechanism combines a highly deformable element with a cable, being possible to change either the cable or the beam lengths, thus, obtaining a larger workspace. In order to solve the kinematics of the robot, the fundamentals of non-linear analysis of flexible bars are briefly explained. The kinematic problem of the parallel continuum robot is then solved using an analytical procedure. To validate the results a prototype has been built in which the beam deformation and the tension suffered by the cable have been measured. 


\section{Fundamentals of Nonlinear Analysis of Flexible Bars}

In the following we will state some fundamentals about the analytical solution of the non-linear deflection of a slender element under bending in a plane. To start with, it acquires the form of a planar curve as in Fig. 1, Navier-Stokes hypothesis assumes that its cross sections remain planar and perpendicular to the bent curve, and Bernoulli-Euler law establishes that the bending moment $M$ at a point is proportional to the curvature $\kappa$ :

$$
\kappa=\frac{d \theta}{d s}=\frac{M}{E I}
$$

where $E$ is the elastic modulus and $I$ is the moment of the cross section about the neutral axis.

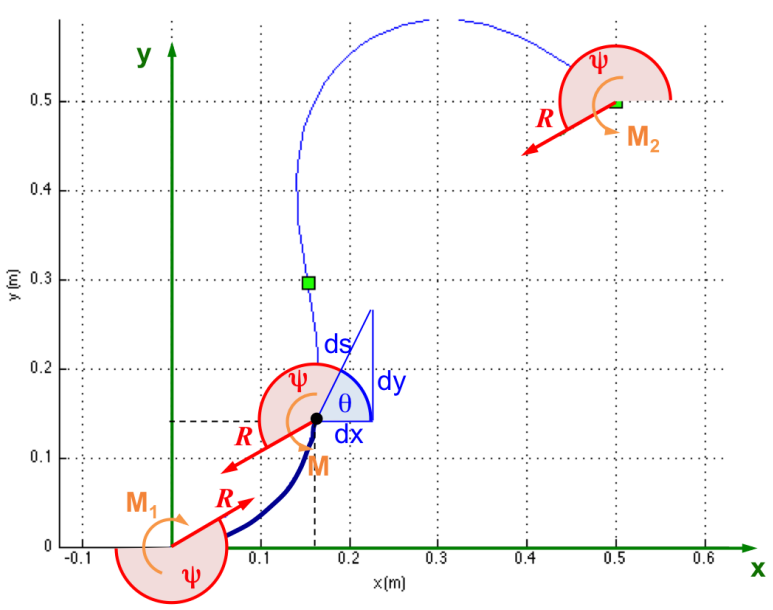

Fig. 1: A section of the bar at equilibrium

We define for a section in equilibrium (see Fig. 1): $R$ and $\psi$ as the reaction force's magnitude and direction at the extreme, $M_{1}$ and $M_{2}$ as the bending moments at a extremes, and $M$ as the bending moment at a cross-section. The static equilibrium of moments for a portion of the bar can be expressed to get $M$ and substitute into Eq. 1:

$$
\kappa=\frac{d \theta}{d s}=\frac{M}{E I}=\frac{M_{1}}{E I}+\frac{R}{E I} \cos \psi y-\frac{R}{E I} \sin \psi x
$$

Its derivative with respect to the arc length $s$, expressed in terms of $\theta$, yields: 


$$
\begin{aligned}
\frac{d \kappa}{d s}=\frac{d^{2} \theta}{d^{2} s} & =\frac{R}{E I} \cos \psi \frac{d y}{d s}-\frac{R}{E I} \sin \psi \frac{d x}{d s}= \\
& =\frac{R}{E I} \cos \psi \sin \theta-\frac{R}{E I} \sin \psi \cos \theta=\frac{R}{E I} \sin (\theta-\psi)
\end{aligned}
$$

Its integration requires a complex mathematical manipulation. Several approaches exist in the literature, here we will follow [4], where we get an integral from one extreme of the bar of length $L$ to the other as:

$$
\sqrt{\frac{R L^{2}}{E I}}=\int_{\phi_{1}}^{\phi_{2}} \frac{1}{\sqrt{1-k^{2} \sin ^{2} \phi}} d \phi=F\left(k, \phi_{2}\right)-F\left(k, \phi_{1}\right)
$$

being $F(k, \phi)$ the incomplete elliptic integral of the first kind, and $k$ and $\phi$ are some auxiliary variables for integration. And the curvature at each point is given by:

$$
\kappa=\frac{d \theta}{d s}=2 k \sqrt{\frac{R}{E I}} \cos \phi
$$

If we are interested in the case of a bar with a clamped end and the other pinned, boundary condition on the slope of the bar at the first extreme, $\theta_{1}$, is given (and always can be taken null), while the other end has a null curvature. For a given force at extremes $R$ and $\psi$, we can state the limits for integration, $\phi_{1}$ and $\phi_{2}$ :

$$
\phi_{1}=\arcsin \left(\frac{1}{k} \cos \left(\frac{\psi}{2}\right)\right) \quad \phi_{2}=q \pi / 2
$$

where $\phi_{1}=[-\pi / 2, \pi / 2]$, and $q$ has even values that determine the Mode of buckling (see Fig. 2). The angle $\phi$ will vary continuously from $\phi_{1}$ to $\phi_{2}$. Then, modulus $k=[-1,1]$ can be obtained iteratively on Eq. (4) for a certain value of $q$. Inflection points of the bar correspond to values $\phi=n \pi / 2$ with even values of $n$ below $q$ if they exist.

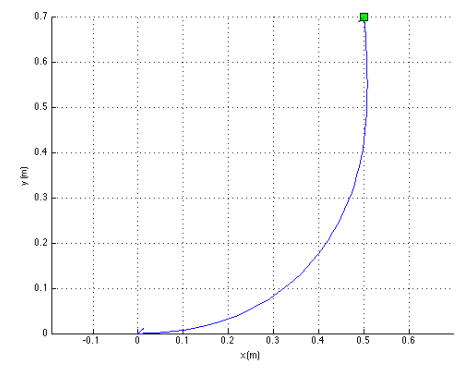

(a)

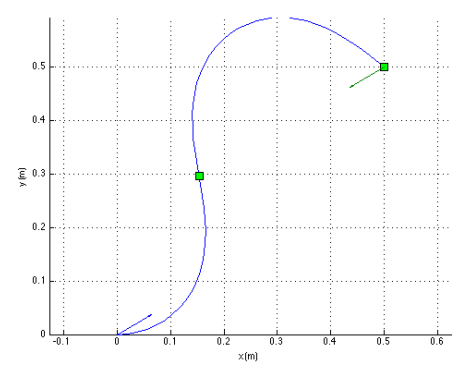

(b)

Fig. 2: Modes 1 and 2 of the buckling of a clamped-pinned bar 
In order to get the $x$ coordinate of a point in the curve we can manipulate Eq. (5) to get the integral:

$$
\begin{aligned}
x= & -\sqrt{\frac{E I}{R}} \cos \psi\left[2 E\left(k, \phi_{i}\right)-2 E\left(k, \phi_{1}\right)-F\left(k, \phi_{i}\right)+F\left(k, \phi_{1}\right)\right]+ \\
& +\sqrt{\frac{E I}{R}} 2 k \sin \psi\left[\cos \phi_{i}-\cos \phi_{1}\right]
\end{aligned}
$$

where $E(k, \phi)$ is the incomplete elliptic integral of the second kind. In order to get the $y$ coordinate an analogous deduction to the one followed for $x$ can be done.

For a given value of the coordinates of the extreme of the deflected bar, i.e. $a, b$, and the boundary conditions, we must iterate on $\psi$ and $k$ in Eq. (4), obtaining $a$ and $b$ from Eqs. (7) and analogous, and verifying that the error obtained is below a given threshold.

From the above results we can infer that solutions can be found between some limiting values for $k$ in an unknown range of $\psi$ and for each mode separately. A minimum value for $k$, upon analysis of Eq. (6), corresponds to $k_{\min }=\left\|\cos \left(\frac{\psi}{2}\right)\right\|$, positive for the range $k=\left[k_{\min }, 1\right]$ and negative for the range $k=\left[-k_{\min },-1\right]$.

If we restrict ourselves to positive values of $k$, and plot the end positions of the bar for a given value of $\psi$ in the range from $k_{\min }$ to $k=1$ we get the plot in Fig. 3 . As it can be seen, solutions for both modes start from the limiting curve of $k_{\min }$ and go up to a $k$ value of 1 .

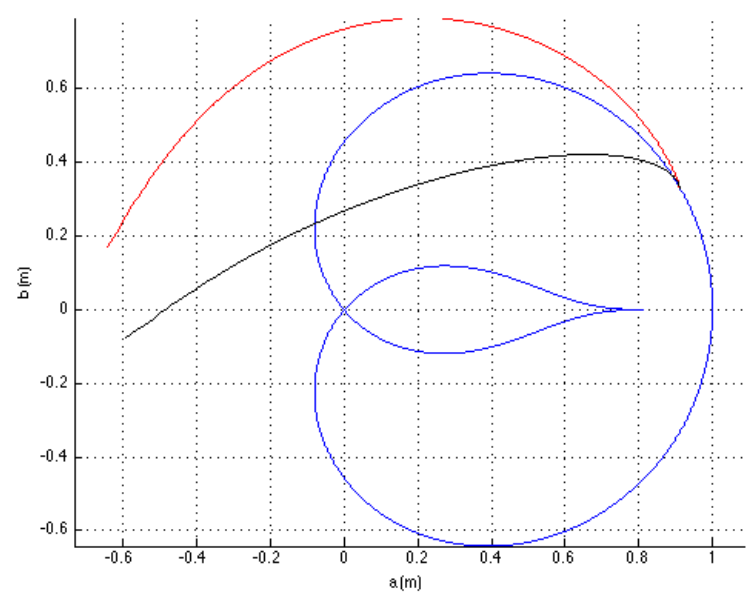

Fig. 3: Solutions for Modes 1 (red curve) and 2 (black curve) for $\psi=200$ from $k_{\text {min }}$ to $\mathrm{k}=1$ 


\section{Kinematic Analysis of the 2 DoF Parallel Continuum Robot}

The mechanism proposed is a closed loop device, see Fig. 4. A slender bar is actuated through a fixed support that keeps a constant orientation at that section, so the length $l$ of the deformed bar is variable. Also, a cable is attached to the extremity of the bar in $P$ and its length $\lambda$ can also be controlled varying $\delta$. Hence, the system has 2 degrees of freedom that control the position on the plane of the end-point $P$.

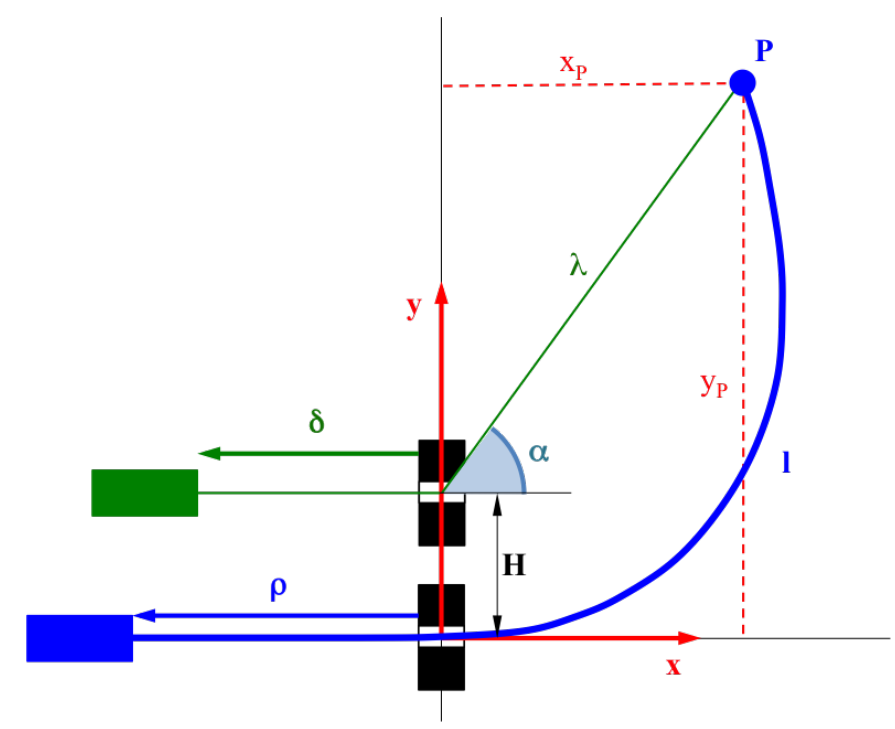

Fig. 4: Continuum Parallel Robot

In order to solve the inverse position problem, given the desired coordinates for $P$, i.e. $x_{P}$ and $y_{P}$, we can find the required length for the cable straightforward. The orientation of the end-force $R$, i.e. the angle $\psi$ of the force applied at the end-point is found. Then, we can iterate in the $k$ parameter for equations Eq. 6 and Eq. 7 until we find a value that produces an end-point position closer to the objective than a given threshold. The analytical solution is quite simple and the iterative process reaches a solution quickly.

The described methodology has been used to obtain information regarding the workspace of the manipulator. The tension of the cable, the Von Mises stress in the exit point of the beam have been calculated. As the analytical procedure solves the inverse kinematic problem, the error between the objective position and the obtained position has also been calculated. All these values can be seen in Fig. 5.

Cable tension and Von Mises stress behave as expected, increasing as deformation in the beam increases. The same happens with the error, as it becomes higher with as the location of the end effector requires a bigger deformation of the beam. 


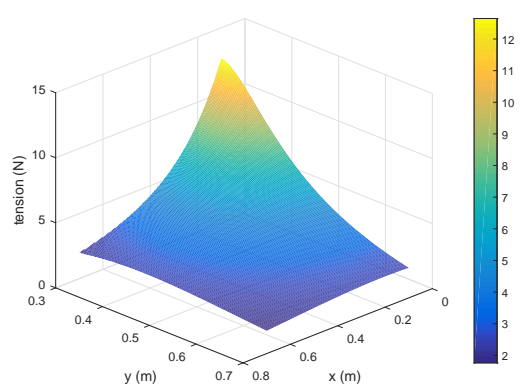

(a)

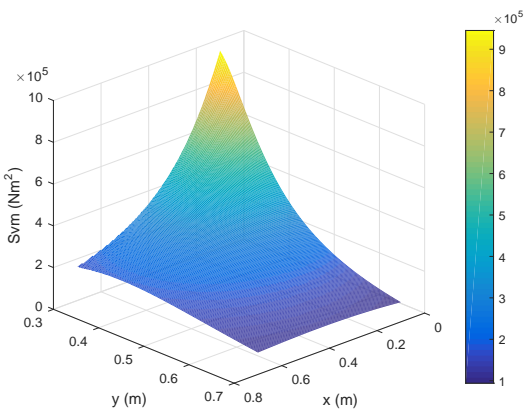

(b)

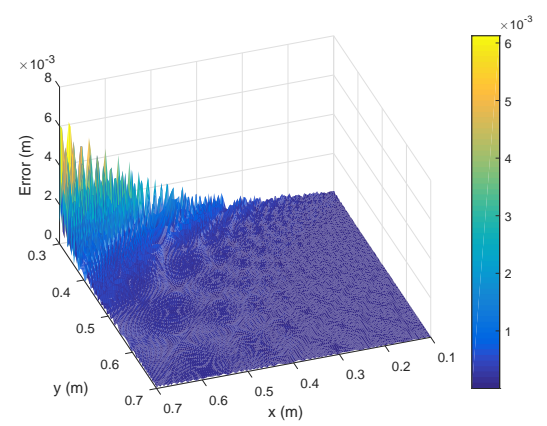

(c)

Fig. 5: Study of the workspace of the manipulator. (a) force in the cable. (b) Von Mises stress in the beam. (c) Analytical procedure error.

\section{Numerical and Experimental Validation}

In order to verify the analytical model of the parallel robot a prototype has been built. In the prototype it is possible to measure the deformation of the beam and the tension in the cable. Measurements have been made using a MC 850 ZEISS threedimensional measuring machine (precision is $+-0.005 \mathrm{~mm}$ ). The experimental setup can be seen in Fig. 6. Two different positions have been measured, corresponding to $x=556.7 \mathrm{~mm} y=335.3 \mathrm{~mm}$ and $x=650.4 \mathrm{~mm} y=199.8 \mathrm{~mm}$.

The numerical approach based on FEM has only been used to derive the direct kinematic problem. The model consists of two elements where the thinner represents the cable under tension and, the bigger one, the element of the model which is subject to bending. Both the elements have been considered as two beams, the cable is a bi articulated beam so it behaves as the cable and the other is a cantilever beam with the free end attached to the cable. The software used in the simulation has been ANSYS ${ }^{\circledR}$. 


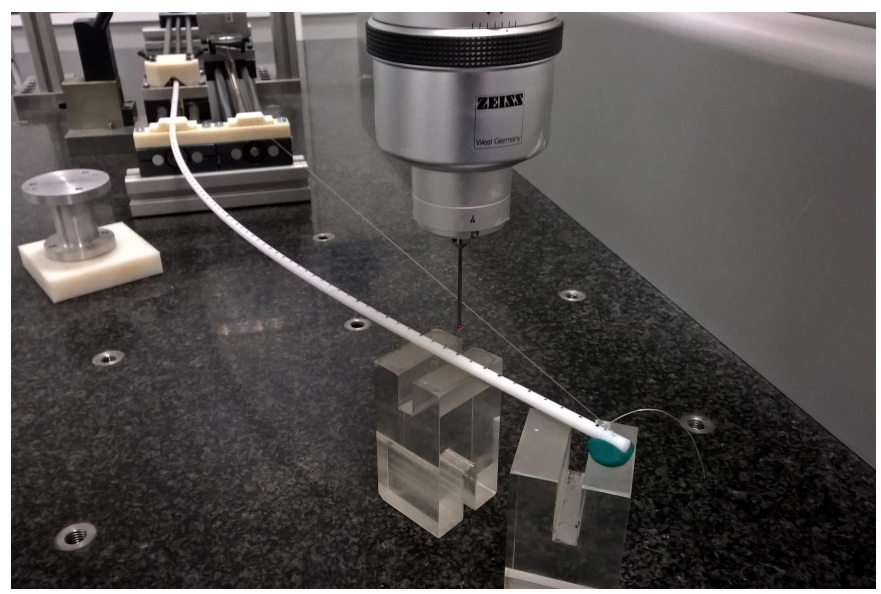

Fig. 6: Experimental setup for experimental measurements

Regarding the deformed shape of the robot, analytical, numerical and experimental model results are compared in Fig. 7. The shape of the deformed models match perfectly the experimental data. Calculated cable length also matches the experimental model measures. Discrepancies appear on the applied force. In Fig. 7b, a cable force of $2.94 \mathrm{~N}$ is applied whereas the analytical model predicts a $2.55 \mathrm{~N}$ force. The same happens in Fig. $7 \mathrm{a}$ where $4.9 \mathrm{~N}$ where applied when the analytical model yielded $3.65 \mathrm{~N}$. For this latter case, the value of the force in the cable obtained from the numerical model yields $3.603 \mathrm{~N}$. This deviation may happen because of the experimental error derived from the accuracy of the measuring devices and the friction suffered by the cable and the guide. The force in the cable has been measured by means of a load cell with a precision of $10 \mathrm{~g}$.

\section{Conclusions}

Combination of deformable elements and cables may produce a feasible parallel continuum robot, that still benefit from the compliant mechanism properties. In this paper a methodology to solve the kinematic problem of such mechanism is proposed. The analytical procedure solves the inverse kinematic problem whereas the direct kinematic problem is solved using FEM. The numerical results are validated with an experimental model, showing good correlation between the numerical and experimental data.

Acknowledgements The authors wish to acknowledge the financial support received from the Spanish Government through the Ministerio de Economía y Competitividad (Project DPI201564450-R) and the Regional Government of the Basque Country through the Departamento de 


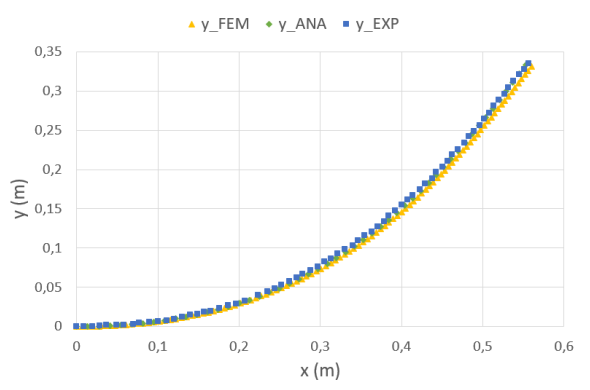

(a)

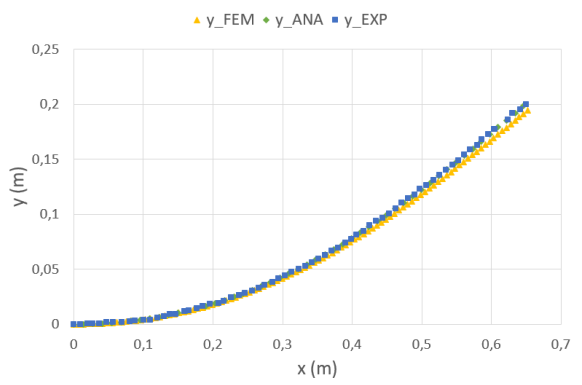

(b)

Fig. 7: Deformed shape comparison for (a) cable force $3.6 \mathrm{~N}$ and (b) cable force $2.55 \mathrm{~N}$.

Educación, Universidades e Investigación (Project IT445-10) and UPV/EHU under program UFI $11 / 29$.

\section{References}

1. Bionic Tripod 3.0. Festo Innovation and Technology. http://www.festo.com

2. Bryson C. E. and Rucker D. C.: Toward Parallel Continuum Manipulators. IEEE International Conference on Robotics and Automation, 778-785, (2014).

3. Antman S.S.: Nonlinear Problems of Elasticity. Springer (2005).

4. Holst, G. L. et al.: Modeling and Experiments of Buckling Modes and Deflection of FixedGuided Beams in Compliant Mechanisms. Journal of Mechanical Design, 133, 051002-1-10 (2011)

5. Zhang, Aimei, and Guimin Chen: A comprehensive elliptic integral solution to the large deflection problems of thin beams in compliant mechanisms. Journal of Mechanisms and Robotics 5, 021006-1. (2013) 